\title{
Transcriptomic coordination in the human metabolic network reveals molecular links between fat intake and metabolic health
}

\author{
M. J. Morine ${ }^{1}$, A. C. Tierney ${ }^{1}$, S. Toomey ${ }_{5}^{1}$, C. Gormley ${ }^{2}$, P. Ó’Gaora ${ }^{3}$, C. A. Drevon ${ }^{4}$, \\ J. Lopez-Miranda ${ }^{5}$ and H. M. Roche \\ ${ }^{1}$ Nutrigenomics Research Group, UCD Conway Institute, University College Dublin, Dublin 4, Ireland, ${ }^{2}$ School of \\ Mathematical Sciences, University College Dublin, Belfield, Dublin 4, Ireland, ${ }^{3}$ UCD School of Medicine and Medical \\ Sciences, UCD Conway Institute, University College Dublin, Dublin 4, Ireland, ${ }^{4}$ Department of Nutrition, Faculty of \\ Medicine, University of Oslo, Norway and ${ }^{5}$ Lipid and Atherosclerosis Unit, Department of Medicine, Reina Sofia University \\ Hospital, School of Medicine, University of Cordoba, CIBER Physiopathology of Obesity and Nutrition (CB06/03), Spain
}

Understanding the functional relationships between diet, gene expression and phenotype is a key challenge in nutritional science. The LIPGENE project was an international research effort assessing the impact of genotype and dietary fat intake on metabolic health ${ }^{(1)}$. In the final stage of this project, we generated adipose tissue microarray data in order to examine the effect of habitual dietary components on transcriptomic activity and, subsequently, markers of metabolic health.

A sub-cohort of participants in the LIPGENE human intervention study were included in this analysis of adipose tissue transcriptomic signatures $^{(2)}$. Habitual dietary data included SFA, MUFA, $\omega-3$ and $\omega-6$ PUFA, carbohydrates, protein and alcohol. Blood samples were collected and assessed for 46 markers of metabolic and cardiovascular health including cholesterol, TAG, insulin sensitivity and glucose metabolism (assessed by IVGTT) and markers of inflammation and oxidative stress. Plasma fatty acid composition was also determined as a marker of dietary fat intake, and urinary 8-iso-PGF2a as a marker of oxidative stress.

As an alternative to pathway analysis, we have taken a network-based approach to the analysis of dietary, transcriptomic and comprehensive plasma marker profile. Sparse partial least squares and regularised canonical correlation analyses were used to identify strongly correlated diet-gene and plasma marker-gene pairs, which were then mapped to a high-quality reconstruction of the human metabolic network. Diet-sensitive and transcriptionally co-expressed sub-paths were subsequently extracted from the network using an algorithm designed to trace paths of enzymatic metabolite conversion.

With this approach we identified a prominent sub-path in the network that was transcriptionally correlated with dietary $\omega-3$ PUFA intake and strongly co-expressed within the sub-path. This sub-path interlinked the related processes of glycerophospholipid, arachidonic acid and linoleic acid metabolism - and was shown to correlate with plasma markers of metabolic health including plasma DHA and urinary 8-9-iso-PGF2a. Promoter analysis further revealed a number of significantly over-represented adipogenic transcription factors (such as pparg and klf4) in the promoter regions of genes that were positively correlated with $\omega$-3 PUFA intake. This integrated bioinformatics approach highlighted novel regulatory and metabolic mechanisms relating dietary $\omega$-3 PUFA intake to metabolic health via adipogenic transcription factors and interconnected lipid metabolism pathways.

This work was co-funded by NuGO and LIPGENE within Framework 6 Food Safety and Quality Programme. M. J. M. is funded by an Irish Research Council for Science, Engineering \& Technology PhD studentship. H. M. R. is the recipient of an SFI Funded Principal Investigator Programme.

1. Buttriss J \& Nugent A (2005) LIPGENE: an integrated approach to tackling the metabolic syndrome. Proc Nutr Soc 64(3), 345-347.

2. Tierney AC, McMonagle J, Shaw DI et al. (2010) Effects of dietary fat modification on insulin sensitivity and other risk factors of the metabolic syndrome - LIPGENE: an European randomised dietary intervention study. Int J Obes (In the Press). 